\section{Editorial}

Check for updates

\title{
Normalization of Cardiac Measurements: Isometric vs. Allometric Method
}

\author{
Sung Hye Kim $(\mathbb{D}, \mathrm{MD}$
}

Department of Pediatrics, CHA Bundang Medical Center, CHA University, Seongnam, Korea

- See the article "Scaling Echocardiographic Cardiac Dimensions to Body Size: A Bayesian Analysis in Healthy Men and Women" in volume 28 on page 10.

Echocardiography is the most commonly used noninvasive modality providing realtime images of the heart. To provide optimal information for decision making, normalization of cardiac measurements is both imperative and clinically significant. For this reason, echocardiography societies have suggested a standardized, formal report of echocardiography, including measurements that are normalized to body size. ${ }^{1)}$

Most studies have published normal values and assume a linear isometric relationship between cardiac dimension and body size, especially body surface area (BSA). The recommendations for cardiac chamber quantification by the American Society of Echocardiography and the European Association of Cardiovascular Imaging provided normal reference values for size and function of both ventricles and size of both atria. ${ }^{2)}$ In this recommendation, normal reference values for left ventricle (LV) volume and LV volume normalized to BSA extracted from databases of normal subjects were provided for men and women. Also, the BSA-indexed LV end diastolic volume (EDV) and end systolic volume (ESV) measured from an apical four-chamber view and the BSA-indexed biplane LV EDV and ESV based on age were shown. Normal value for left atrium (LA) size was also adjusted for body size, especially BSA, for which there is the most available data. ${ }^{34)}$ In addition, the sex differences in LA size are generally accounted for when adjusting for BSA. ${ }^{3)}$ Additionally, the most commonly accepted guideline for the tricuspid annulus (TA) size defines the normal TA diameter in adults as $2.8 \pm 0.5 \mathrm{~cm}$ in the apical four chamber view, and significant TA dilation is defined by a diastolic diameter $>2.1 \mathrm{~cm} / \mathrm{m}^{2}$. 5

On the other hand, this approach with BSA to the power of 1 cannot always explain the relationships between cardiac measurements and body size, and there have been trials to normalize cardiac measures with allometric analysis. De Simone et al. ${ }^{6}$ aimed to identify the optimal method to normalize LV mass (LVM) for body size in overweight children and adults. In his study, LVM was related to BSA to the power of 1.5 and height to the power of 2.7. Normalizations of LVM for height or BSA in his study could not expect exact relations of indexed LVM to body size and caused errors in estimating the impact of overweight. Also, he suggested that LV hypertrophy (LVH) should be identified by LVM normalized by body size raised to allometric powers, such as LVM/BSA ${ }^{1.5}$ or $\mathrm{LVM} / \mathrm{m}^{2.13}$. $^{7)} \mathrm{LVH}$ for $\mathrm{LVM} / \mathrm{BSA}^{1.5}$ or $\mathrm{LVM} / \mathrm{m}^{2.13}$ was detected more frequently than $\mathrm{LVH}$ for BSA and was associated with a larger 
proportion of incident cardiovascular events. In addition, this approach allows detection of adverse obesity-related LVH. Normalizing cardiac chamber measurements with allometric scaling methods in obese patients was also suggested in another study by Zong et $\mathrm{al}^{8}$ who analyzed left chamber measurements in 717 healthy obese adults to body size variables, such as height, weight, body mass index, and BSA, allometric scaling of LA anteroposterior dimension (LAD), LA volume, LVEDV, and LV end diastolic dimension showed stronger correlation with measured chamber sizes compared with isometric scaling, and the overcorrection caused by isometric scaling was overcome with allometric measurements. These studies imply that isometric measurements, such as cardiac measurements relative to height or BSA, would be misleading, especially in overweight and obese subjects. Neilan et al. ${ }^{9)}$ compared isometric and allometric modeling approaches to assess the optimal scaling method using LAD with 15,667 consecutive echocardiographic studies for normal adults. In his study, the standard isometric corrections for body size failed to adequately account for the association between LAD and body size. Normalization using the optimal allometric exponent removed the effect of body size, especially that of body weight.

However, such results are not always consistent, and allometric coefficients (AC) are variable. A study by Daniels et al. ${ }^{10)}$ revealed height ${ }^{3}$ as the optimal height exponent for indexing LVM in children and adolescents, whereas Chirinos et al. ${ }^{11)}$ reported LVM/height ${ }^{1.7}$ as more sensitive than LVM/BSA to identify obesity-related LVH and predict cardiovascular events in adults. These results suggest the age effect on the allometric signal of height.

In this issue of Journal of Cardiovascular Imaging, Iglesias-Garriz et al. ${ }^{12)}$ tried to identify the allometric relationships between cardiac measurements and body size. In measurements of LVEDV, LVESV, and TA, AC did not coincide with the isometric coefficient, and AC for LVEDV and LVESV were 1.5 and 1.7, respectively, which indicates that normalization using $\mathrm{BSA}^{1.5}$ and $\mathrm{BSA}^{1.7}$ could produce a better scaling of these volumes. However, in the study, the sample size was not large enough, and this limitation hindered them from considering the effects of age, sex, or other possible factors on body size in their analyses.

The allometric approach is not a formal recommendation in most guidelines. Application of the allometric approach for normalization of cardiac chamber measurements requires further study in a large population with a wide range of age and body size, including obesity.

\section{REFERENCES}

1. Galderisi M, Cosyns B, Edvardsen T, et al.. Standardization of adult transthoracic echocardiography reporting in agreement with recent chamber quantification, diastolic function, and heart valve disease recommendations: an expert consensus document of the European Association of Cardiovascular Imaging. Eur Heart J Cardiovasc Imaging 2017;18:1301-10. PUBMED | CROSSREF

2. Lang RM, Badano LP, Mor-Avi V, et al. Recommendations for cardiac chamber quantification by echocardiography in adults: an update from the American Society of Echocardiography and the European Association of Cardiovascular Imaging. J Am Soc Echocardiogr 2015;28:1-39.e14. PUBMED | CROSSREF

3. Kou S, Caballero L, Dulgheru R, et al. Echocardiographic reference ranges for normal cardiac chamber size: results from the NORRE study. Eur Heart J Cardiovasc Imaging 2014;15:680-90. PUBMED | CROSSREF

4. Pritchett AM, Jacobsen SJ, Mahoney DW, Rodeheffer RJ, Bailey KR, Redfield MM. Left atrial volume as an index of left atrial size: a population-based study. J Am Coll Cardiol 2003;41:1036-43.

PUBMED | CROSSREF 
5. Lancellotti P, Moura L, Pierard LA, et al.. European Association of Echocardiography recommendations for the assessment of valvular regurgitation. Part 2: mitral and tricuspid regurgitation (native valve disease). Eur Echocardiogr 2010;11:307-32.

PUBMED | CROSSREF

6. de Simone G, Daniels SR, Devereux RB, et al. Left ventricular mass and body size in normotensive children and adults: assessment of allometric relations and impact of overweight. J Am Coll Cardiol 1992;20:1251-60.

PUBMED | CROSSREF

7. de Simone G, Kizer JR, Chinali M, et al.. Normalization for body size and population-attributable risk of left ventricular hypertrophy: the Strong Heart Study. Am J Hypertens 2005;18:191-6. PUBMED | CROSSREF

8. Zong P, Zhang L, Shaban NM, Peña J, Jiang L, Taub CC. Left heart chamber quantification in obese patients: how does larger body size affect echocardiographic measurements? J Am Soc Echocardiogr 2014;27:1267-74.

PUBMED | CROSSREF

9. Neilan TG, Pradhan AD, Weyman AE. Derivation of a size-independent variable for scaling of cardiac dimensions in a normal adult population. J Am Soc Echocardiogr 2008;21:779-85.

PUBMED | CROSSREF

10. Daniels SR, Kimball TR, Morrison JA, Khoury P, Meyer RA. Indexing left ventricular mass to account for differences in body size in children and adolescents without cardiovascular disease. Am J Cardiol 1995;76:699-701.

PUBMED | CROSSREF

11. Chirinos JA, Segers P, De Buyzere ML, et al. Left ventricular mass: allometric scaling, normative values, effect of obesity, and prognostic performance. Hypertension 2010;56:91-8.

PUBMED | CROSSREF

12. Iglesias-Garriz I, Alonso D, Garrote C, et al. Scaling echocardiographic cardiac dimensions to body size: a Bayesian analysis in healthy men and women. J Cardiovasc Imaging 2020;28:10-7.

PUBMED | CROSSREF 